\title{
FATORES AMBIENTAIS SOBRE A IDADE AO PRIMEIRO PARTO, DIAS ABERTOS E INTERVALO ENTRE PARTOS EM VACAS DA RAÇA HOLANDESA NA BACIA LEITEIRA DE CASTROLANDA, ESTADO DO PARANÁ
}

(Environmental factors that influence the age at the first calving, days open and calving interval in Holstein cows of the Castrolanda, State of Paraná)

\author{
WOLFF, M.C.C. ${ }^{1}$; MONARDES, H.G. ${ }^{2}$; RIBAS, N.P. ${ }^{3}$
}

${ }^{1}$ Méd. Vet., Mestra em Ciências Veterinárias pela Universidade Federal do Paraná;

${ }^{2}$ M. Sc.; Ph.D. - Department of Animal Science, McGill University;

${ }^{3}$ Med. Vet., M. Sc. - Professor Adjunto N-4 do Departamento de Zootecnia, Universidade Federal do Paraná.

\begin{abstract}
RESUMO - Dados provenientes do Programa de Análise de Rebanhos Leiteiros do Paraná (PARLPR) da Associação Paranaense de Criadores de Bovinos Leiteiros da Raça Holandesa (APCBRH) foram analisados para estudar os fatores do meio ambiente (rebanho, ano de parto, mês de parto, grupo genético, idade ao parto e efeito vaca) que estariam influenciando as características reprodutivas: idade ao primeiro parto (IPP) em meses, dias abertos (DA) e intervalo entre partos (IEP) em dias, nas vacas da raça Holandesa na Bacia Leiteira de Castrolanda, Castro, Estado do Paraná. Para o estudo dos efeitos de meio ambiente sobre a idade ao primeiro parto, foram utilizadas 10.494 primíparas da raça Holandesa, variedade HPB (Preta e Branca), pertencentes a 68 rebanhos, controlados entre 1991 e 2000. Para os dias abertos e intervalo entre partos, foram utilizadas 16.232 vacas, pertencentes a 67 rebanhos, das mesmas raça e variedade. As médias e os respectivos desvios-padrão observados para o IPP, DAe IEP, foram: 27,05 $\pm 3,93$ meses, 98,73 $\pm 33,03$ dias e $380,73 \pm 33,03$ dias, respectivamente. Para as análises estatísticas dos dados foi utilizado o Método GLS (General Least Square), pelo Proc Mixed do programa SAS ${ }^{\circledR}$, versão 6.1. Os efeitos de rebanho, ano de parto, mês de parto e grupo genético foram altamente significativos $(P<0,01)$ sobre a IPP. Os efeitos de rebanho, ano de parto, mês de parto e idade ao parto foram altamente significativos $(P<0,01)$ e o grupo genético, não significativo, para DA e IEP. As médias observadas para as três características foram inferiores às relatadas em outras regiões do Brasil, equiparando-se às médias das bacias leiteiras dos países com bovinocultura leiteira mais desenvolvida.
\end{abstract}

Palavras chave: Holandesa, parto, dias abertos.

ABSTRACT - Data of the Programa deAnálises de Rebanhos Leiteiros do Paraná (PARLPR) of the Associação Paranaense de Criadores de Bovinos da Raça Holandesa (APCBRH) were analyzed, to study the environmental factors (herd, year of calving, calving month, genetic group, age of calving and cow effect) that influence the reproduction traits: age at the first calving in months, days open and calving interval, in days, cows in Castrolanda, Castro, State of Paraná. To study the age at the first calving, 10,494 Black-and-White Holstein heifers, from 68 herds, were used, being controlled between 1991 and 2000. For days open and calving interval, 16,232 cows were used, from 67 herds. The averages observed with the respective standard deviations for age at the first calving, days open and calving interval, were: $27,05 \pm 3,93$ months, $98,73 \pm 33,03$ days and $380,73 \pm 33,03$ days, respectively. For the statistical analyse of the data, the GLS (General Least Squares) method was used, by Proc Mixed of the program $S A S^{\circledR}$, version 6.1. The effects of herd, year of calving, calving month and genetic group were highly significant $(P<0,01)$ about the age at the first calving. The effects of herd, year of calving, calving month and age at calving were highly significant $(P<0,01)$ and the genetic group, not significant, for days open and calving interval. The averages observed for the three characteristics were lower than the reported in others Brazilian states, and they are close to the averages of the milk yield from developed countries.

Key words: Holstein, calving, days open.

Correspondência para: WOLFF, M.C.C. - Rua Arthur Thomas, 410 - Centro - Maringá / Pr - 87013-250. Email:mccw@pr.gov.br. 


\section{Introdução}

A pecuária nacional convive há quase um século com baixa produtividade. Com isso, o retorno econômico fica muito aquém do potencial da atividade. A produção de leite no Brasil elevouse de quase 5 bilhões de litros/ano em 1960, para os atuais 21 bilhões. Esse acréscimo de 16 bilhões de litros em 39 anos ocorreu mais pelo aumento no número de vacas ordenhadas que pelo aumento de produtividade (ANUÁRIO, 2002).

A baixa produtividade dos rebanhos leiteiros nacionais seja na produção de leite pela unidade de área (litros/hectare/ano) ou média de produção por vaca/ano, deve-se essencialmente aos seguintes fatores: mau desempenho reprodutivo e inferior qualidade genética dos animais nos quesitos produção, duração e persistência da lactação (FARIA, 2002).

As características idade ao primeiro parto, dias abertos e intervalo entre partos são medidas práticas, que traduzem a eficiência reprodutiva dos rebanhos. A idade avançada ao primeiro parto, dias abertos e intervalos entre partos longos, reduzem a produção vitalícia dos animais e, conseqüentemente, diminuem a lucratividade da atividade leiteira, segundo RIBAS et al. (1997a).

A avaliação dos efeitos de meio ambiente é uma importante parte dos estudos de performance, em se tratando de gado de leite. Muitas vezes, problemas de adaptação ao meio ambiente e o clima adverso da região tropical poderá constituir fator limitante ao desempenho do animal, uma vez que as principais raças leiteiras originam-se de regiões temperadas $e$ as maiores pressões de seleção ocorreram em tais condições.

Assim, este estudo teve por objetivos verificar os fatores do meio ambiente (rebanho, ano de parto, mês de parto, grupo genético, idade ao parto e efeito vaca) que estariam influenciando a idade ao primeiro parto, o intervalo entre partos e os dias abertos das vacas da raça holandesa na Bacia Leiteira de Castrolanda, Castro, Estado do Paraná, em rebanhos sob controle oficial no período de 1991 a 2000.

\section{Material e Métodos}

Os dados utilizados neste estudo foram provenientes do banco de dados do Laboratório
Centralizado de Análise de Leite, do Programa de Análise de Rebanhos Leiteiros do Paraná (PARLPR).

As restrições impostas para a seleção e conseqüente exclusão dos dados foram: raça não holandesa; ano de parto anterior a 1991; idade ao parto inferior a 20 e superior a 144 meses; idade ao primeiro parto inferior a 20 e superior a 42 meses; dias abertos inferiores a 40 e superiores a 365 dias e intervalo entre partos inferior a 322 e superior a 450 dias.

As análises estatísticas dos dados foram efetuadas com a utilização do pacote estatístico SAS $®$, versão 6.1 (SAS® System for Linear Models, 1991), através dos procedimentos PROC MEANS, PROC FREQ, PROC REG e PROC MIXED (Método GLS -General Least Square).

No modelo estatístico para a característica idade ao primeiro parto, foram utilizados os efeitos de rebanho (68, com 10.494 observações), ano de parto (1991 a 2000), mês de parto (janeiro a dezembro) e grupo genético. Os grupos genéticos foram organizados em nove classes (de 1 a 9) expressas na TABELA 1.

Nos modelos estatísticos para as características dias abertos e intervalo entre partos, foram utilizados os efeitos de rebanho (67, com 16.232 observações), ano de parto (1991 a 2000), mês de parto (janeiro a dezembro), grupo genético (classes de 1 a 9), idade ao parto, coeficientes de correlação linear (b ou b ${ }^{1}$ ) e efeito vaca (8.370). A idade ao parto foi dividida em classes (de 1 a 5 ), expressas na TABELA 2.

\section{Resultados e Discussão}

Idade ao Primeiro Parto: A média da idade ao primeiro parto, foi de 27,0 , significando que as novilhas foram cobertas e tornaram-se prenhas aos 18 meses aproximadamente.

Segundo o Programme d'Analyse des Troupeaux Laiteiers du Québec-PATLQ (2001), no Canadá, a idade ao primeiro parto ideal seria de 24 a 25 meses, sobre a qual haveria maior aproveitamento da vida produtiva do animal, muito embora SIMERL et al. (1992), relatam que novilhas muito precoces geralmente apresentam problemas de parto e a produção acaba sendo inferior à média. Por outro lado, a novilha muito 
Fatores ambientais sobre a idade ao primeiro parto, dias abertos e intervalo entre partos em vacas da raça...

tardia tem o aproveitamento da vida produtiva reduzido. Valores superiores à média encontrada nesse trabalho foram verificados, no exterior, por HOLMANN et al. (1990), na Venezuela, de 34,0 meses, e por ZARNECKI et al. (1991), na Polônia, de 29,1 meses. No Brasil, há diversos estudos realizados nas últimas duas décadas, nos quais verificou-se valor superior: BASILE et al. (1986), POLASTRE et al. (1987), RORATO (1988), FREITAS et al. (1991), RICHTER (1995), ALMEIDA (1996) e PIMPÃO (1996), encontraram valores de 38,7, 34,9, 31,0, 36,8, $30,2,30,3$ e 29,4 respectivamente.

TABELA 1 - NÚMERO DE OBSERVAÇÕES (N) POR CLASSES DE GRUPOS GENÉTICOS DA IDADE AO PRIMEIRO PARTO (IPP), DIAS ABERTOS (DA) E INTERVALO ENTRE PARTOS (IEP) EM VACAS DA RAÇA HOLANDESA PRETAE BRANCA. (2004).

\begin{tabular}{lcc}
\hline \multicolumn{1}{c}{ Classe } & IPP $(\mathrm{N})$ & $\mathrm{DA} / \mathrm{IEP}(\mathrm{N})$ \\
\hline 1 (PCOD 31/32 $\Rightarrow$ puro por cruza de origem desconhecida) & 3 & 142 \\
$2(\mathrm{GC}-1 \Rightarrow$ geração controlada 1) & 61 & 74 \\
$3(\mathrm{GC}-2 \Rightarrow$ geração controlada 2) & 373 & 912 \\
$4(\mathrm{GC}-3 \Rightarrow$ geração controlada 3) & 609 & 1382 \\
$5(\mathrm{GC}-4 \Rightarrow$ geração controlada 4) & 790 & 1477 \\
$6(\mathrm{GC}-5 \Rightarrow$ geração controlada 5) & 832 & 1168 \\
$7(\mathrm{GC}-6 \Rightarrow$ geração controlada 6) & 602 & 834 \\
$8(\mathrm{GC}-7 \Rightarrow$ maior ou igual a geração controlada 7$)$ & 705 & 698 \\
$9(\mathrm{PO} \Rightarrow$ puro de origem) & 6519 & 9545 \\
\hline Total & 10494 & 16232 \\
\hline
\end{tabular}

TABELA 2 - NÚMERO DE OBSERVAÇÕES E PORCENTAGENS POR CLASSES DE IDADE AO PARTO EM MESES EM VACAS DA RAÇA HOLANDESA PRETA E BRANCA. (2004).

\begin{tabular}{cccc}
\hline Classe & Idade & $\mathrm{N}^{\circ}$ de Observações & $\%$ \\
\hline 1 & $<=48$ & 6113 & 37,7 \\
2 & $>48 \mathrm{e}<=68$ & 5579 & 34,4 \\
3 & $>68 \mathrm{e}<=88$ & 2726 & 16,8 \\
4 & $>88 \mathrm{e}<=108$ & 1258 & 7,8 \\
5 & $>108$ & 556 & 3,4 \\
\hline Total & & 16232 & 100 \\
\hline
\end{tabular}

O manejo reprodutivo das novilhas da região de Castrolanda mostrou-se bastante eficiente, e se traduziu pela idade ao primeiro parto próxima aos padrões canadenses. Isto foi evidenciado com a divisão das idades em classes, e verificou-se que $42,0 \%$ das primíparas apresentaram o primeiro parto com idade inferior a 25 meses.

Rebanho: A análise de variância mostrou que o efeito de rebanho foi altamente significativo $(P<0,01)$. Este fato demonstra, principalmente, o conjunto de práticas de manejo utilizadas em cada rebanho, na criação e no desenvolvimento de bezerras, com a finalidade de se obter em novilhas que atinjam o peso ideal para a primeira cobertura aos 15-16 meses.
Ano de Parto: O efeito de ano de parto foi altamente significativo. Influência deste efeito também foi observada por RIBAS et al. (1983), RICHTER (1995), MONARDES et al. (1995) e PIMPÃO (1996). No GRÁFICO 1, verifica-se uma tendência de diminuição da idade ao primeiro parto com o passar dos anos. Este resultado sinaliza mudança gradativa no manejo adotado pelos produtores, orientados pelas áreas técnicas, trazendo como conseqüência, precocidade sexual e maior número de lactações na vida reprodutiva dos animais (RICHTER, 1995), possibilitando a antecipação do descarte e favorecendo o rápido melhoramento genético dos rebanhos.

Mês de Parto: O efeito do mês de parto, da mesma forma, apresentou alta significância. A 
maior média foi observada no mês de fevereiro (28,1 meses), sendo que no mês de agosto, foi registrada a menor média, (27,2 meses), caracterizando mudanças estacionais do padrão nutricional (RIBAS et al., 1983; BASILE et al., 1986) e demonstrando que as concepções de outono e inverno são das primíparas mais tardias.

Grupo Genético: O último fator estudado também mostrou-se altamente significativo para a idade ao primeiro parto. Houve uma tendência de redução da idade ao primeiro parto à medida que aumentou o número de gerações controladas, em direção ao puro de origem. $\mathrm{O}$ mesmo foi concluído da pesquisa feita por HOLMANN et al. (1990), na Venezuela, e MONARDES et al. (1995), no Brasil, ao observarem que as novilhas puras foram mais precoces que as de menor grau de pureza.

\section{GRÁFICO 1 - IDADE AO PRIMEIRO PARTO, EM MESES, EM FUNÇÃO DO ANO DE PARTO, EM VACAS DA RAÇA HOLANDESA PRETA E BRANCA.(2004).}

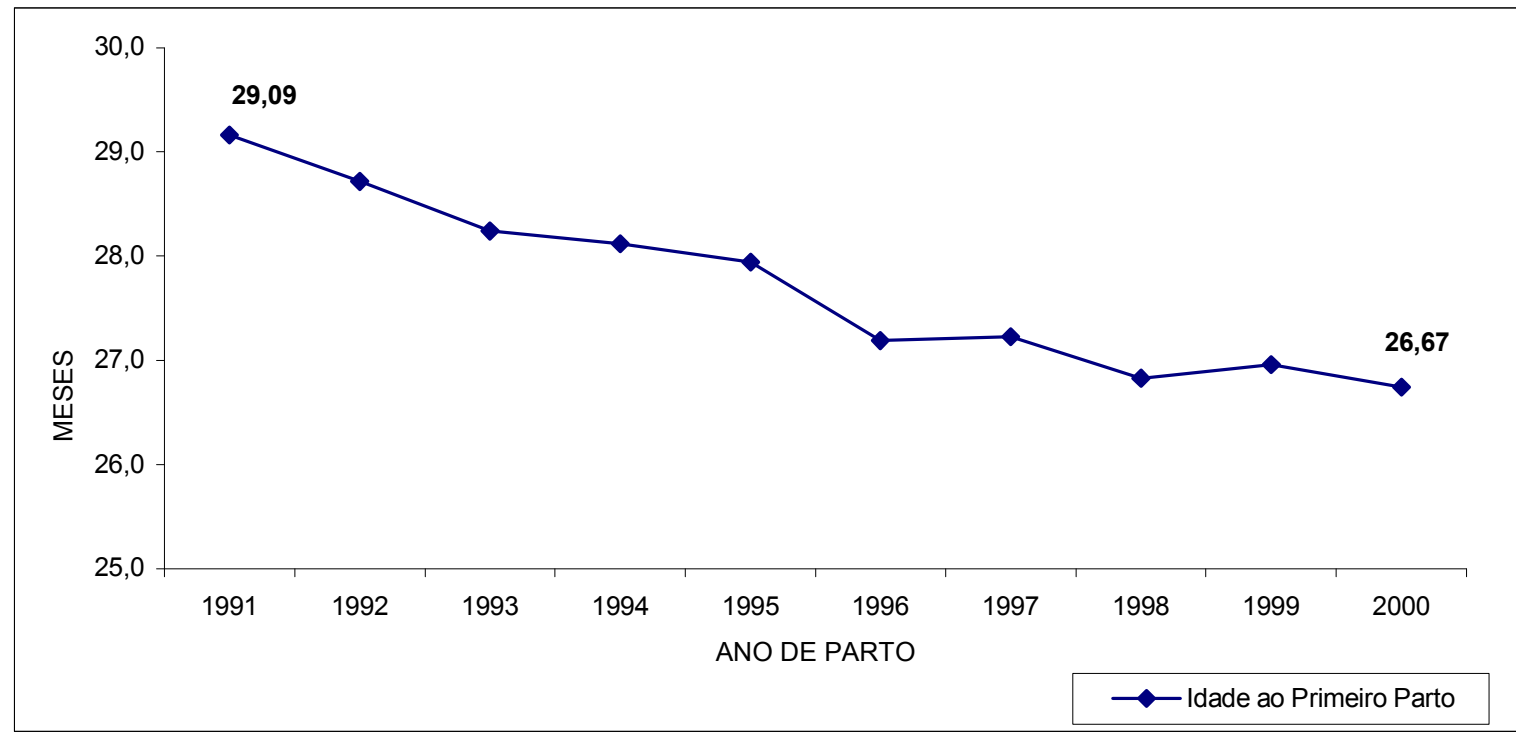

Dias Abertos e Intervalo Entre Partos: A média e o desvio-padrão para a variável dias abertos foi de 98,7 dias e 33,0, respectivamente, para o intervalo entre partos foi, respectivamente, de 380,7 e 33,0 dias. Valores superiores às médias encontradas nesse trabalho foram relatados, no Brasil, por RICHTER (1995), ALMEIDA (1996), PIMPÃO (1996) e PEREIRA et al. (2000) para a variável dias abertos: 116,0, 123,8, 116,0, e 121,2 dias, respectivamente; e por RIBAS et al. (1983), RICHTER (1995), ALMEIDA (1996) e PIMPÃO (1996), para o intervalo entre partos: 408,0, 396,0, 403,8 e 396,0 dias, respectivamente.

Segundo RICHTER (1995), um período de dias abertos ideal situa-se entre 40 e 120 dias; para o intervalo entre partos, é recomendado de 322 a 402 dias. Portanto, os resultados encontrados neste estudo estão dentro do preconizado e demonstram que os criadores da Castrolanda adotam manejos nutricionais e reprodutivos adequados e específicos aos seus rebanhos.
Rebanho: O efeito de rebanho mostrou-se altamente significativo para ambas as características. Resultados semelhantes foram observados por RIBAS et al. (1983), QUEIROZ et al. (1987), RICHTER (1995) e PIMPÃO (1996), no Brasil. Nos Estados Unidos, SILVA et al. (1992), igualmente encontrando efeito significativo, explicaram que isso se justifica pela decisão dos criadores, em relação ao manejo reprodutivo das vacas, na busca da melhor época de cobertura.

Ano de Parto: O ano de parto influenciou significativamente. No GRÁFICO 2, observa-se uma tendência de aumento dos dias abertos e do intervalo entre partos, ao longo dos anos. Isso pode ser conseqüência de que vacas de alta produção, estão sujeitas a distúrbios metabólicos, que podem afetar adversamente a eficiência reprodutiva, apresentando também o próximo estro mais tardiamente do que as de baixa produção além de maior percentual de cios silenciosos (SANTOS, 1998; VÁSQUEZ e HERRERA, 2003). 
Fatores ambientais sobre a idade ao primeiro parto, dias abertos e intervalo entre partos em vacas da raça...

\section{GRÁFICO 2 - DIAS ABERTOS E INTERVALO ENTRE PARTOS (IEP) EM FUNÇÃO DO ANO DE PARTO, EM VACAS DA RAÇA HOLANDESA PRETA E BRANCA. (2004).}

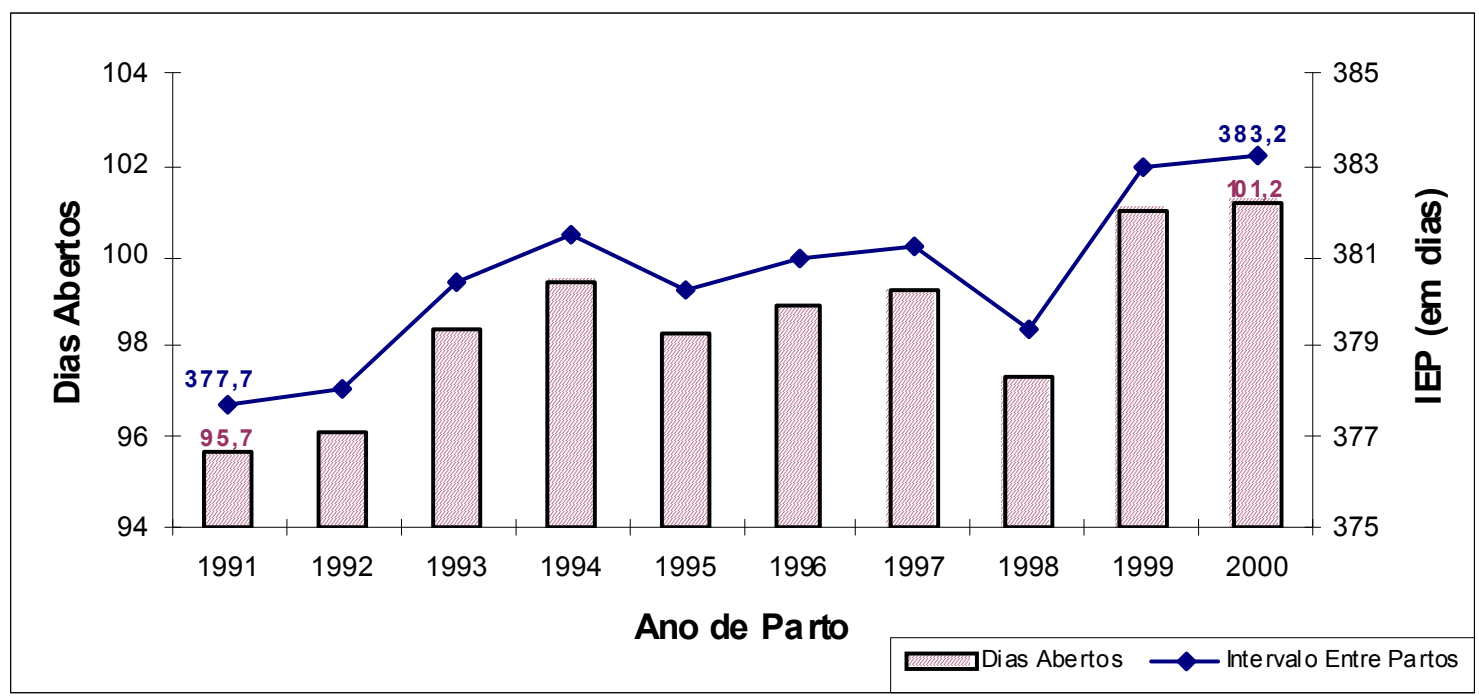

Mês de Parto: O mês de parto teve influência significativa para as duas características. Os menores dias abertos e intervalos entre partos, de 95,3 e 377,3 dias, respectivamente, quando ocorreram as parições ocorreram em abril, e os maiores, de 101,3 e 383,3 dias, quando os partos ocorreram em agosto.

A ocorrência de menores períodos para parições no mês de abril pode ser conseqüência da melhor condição corporal que as vacas apresentam na saída do verão, e, principalmente, devido ao fato de que, nos meses de junho e julho, quando ocorreu a maioria das concepções destes animais, havia maior disponibilidade de pastagens anuais de inverno (RIBAS et al., 1997b). Por outro lado, os menores períodos, quando as parições aconteceram no final do inverno, sugerem desordem nutricional, conseqüência da estiagem que ocorre nessa época no segundo planalto, ou, de desequilíbrio na relação energia / proteína ou excesso de proteína degradável na alimentação, que, em vacas de alta produção, pode causar mortalidade embrionária e queda nas taxas de fertilidade (SANTOS, 1998; VÁSQUEZ e HERRERA, 2003).

Grupo Genético: O efeito do grupo genético não apresentou significância sobre estas características. O fato deste efeito não ter sido significativo decorre de se tratar de um fator atrelado mais à variações de meio ambiente e manejo do que do ponto de vista da constituição genética.

Informações sobre grupo genético são restritas na literatura, tendo em vista que nos países de pecuária leiteira mais desenvolvida, e, por conseqüência, onde há maior disponibilidade de pesquisas, há menor variabilidade de grupos genéticos, pois os rebanhos são constituídos por elevada pureza racial (ALMEIDA, 1996).

Idade ao Parto: $\quad$ O efeito da idade da vaca ao parto mostrou-se altamente significativo no presente estudo.

$\mathrm{Na}$ análise dos coeficientes de regressão apresentada no GRÁFICO 3, evidencia-se um nítido aumento gradativo: os menores valores médios, de 92,5 e 374,4 dias, para dias abertos e intervalo entre partos, respectivamente, nas vacas da classe 1 e os maiores valores, de 104,1 e 386,0 dias, nas vacas com idade classe 5 .

$\mathrm{O}$ aumento do período de dias abertos e de intervalo entre partos, à medida que aumenta a idade das vacas, pode ser decorrente do fato de que vacas adultas apresentam maiores produções do que animais mais jovens (RICHTER, 1995; PIMPÃO, 1996; ANDRADE, 2002), o que pode comprometer os seus desempenhos reprodutivos. Além disso, vacas mais velhas tendem a acumular problemas reprodutivos (ZOCCAL, 2001). 


\section{GRÁFICO 3 - DIAS ABERTOS E INTERVALO ENTRE PARTOS (IEP), EM DIAS, EM FUNÇÃO DA IDADE AO PARTO EM MESES (2004).}

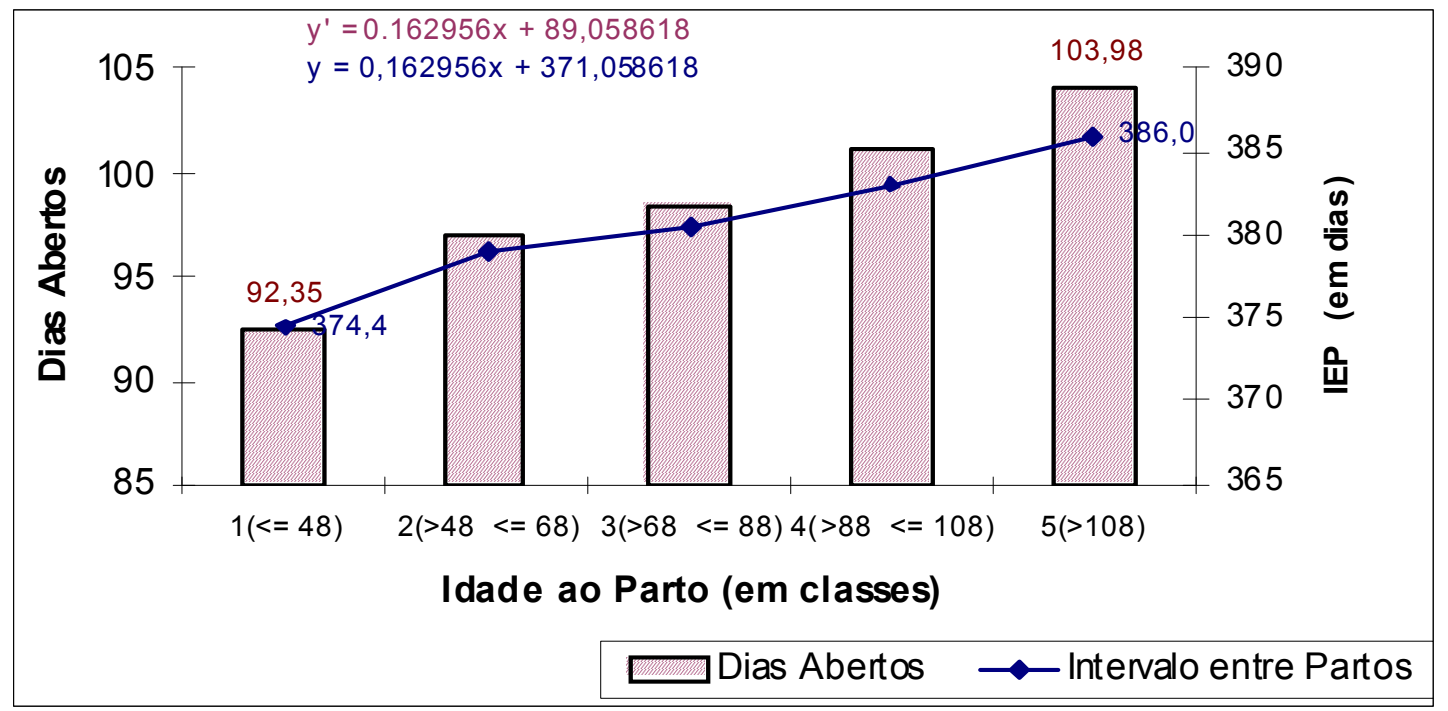

\section{Conclusões}

Os resultados obtidos neste estudo, em condições de ambiente subtropical, permitem concluir que:

Os efeitos de rebanho, ano de parto, mês de parto e grupo genético, foram significativamente menores para a característica idade ao primeiro parto; e os efeitos de rebanho, ano de parto, mês de parto e idade ao parto foram altamente significativos para os dias abertos e o intervalo entre partos.

A idade ao primeiro parto está sendo, gradativamente reduzida, possibilitando a antecipação do descarte e favorecendo o rápido melhoramento genético dos rebanhos.

Com o passar dos anos, há tendência de aumento dos dias abertos, e, conseqüentemente, do intervalo entre partos, sugerindo que vacas de alta produção estão sujeitas a distúrbios metabólicos, que podem afetar adversamente a eficiência reprodutiva.

Aidade ao parto pode afetar significativamente a eficiência reprodutiva da vaca, traduzida por maiores dias abertos e intervalos entre partos à medida que aumenta a maturidade do animal.

\section{Referências}

ALMEIDA, R. Estudo dos efeitos de meio ambiente e genéticos sobre as características produtivas de vacas da raça holandesa na região da Batavo, Estado do Paraná. Curitiba, 1996. 110 F. Dissertação (Mestrado em Ciências Veterinárias) - Setor de Ciências Agrárias, Universidade Federal do Paraná.
ANDRADE, U.V.C. Fatores ambientais sobre a produção total de leite, gordura e proteína em vacas da raça holandesa na bacia leiteira de Castrolanda, Estado do Paraná. Curitiba, 2002. 44 f. Dissertação (Mestrado em Ciências Veterinárias) - Setor de Ciências Agrárias, Universidade Federal do Paraná.

ANUÁRIO de Pecuária 2002. Revista DBO Rural, São Paulo, n.256, mar. 2002.

BASILE, J.R.; RIBAS, N.P.; KOEHLER, H.S. Efeito de meio ambiente na idade ao primeiro parto de um rebanho Holandês (malhado de preto e malhado de vermelho) criado no Estado do Paraná. Revista Brasileira de Reprodução Animal, Belo Horizonte, v.10, n.3, p.137-45, 1986.

FARIA, V.P. Goiânia passa o leite a limpo. Revista DBO Rural, São Paulo, n.255, p.39, 2002.

FREITAS, A.F.; MILAGRES, J.C.; TEIXEIRA, N.M.; CASTRO, A.C.G. Produção de leite em rebanho leiteiro mestiço. Revista da Sociedade Brasileira de Zootecnia, Viçosa, v.20, n.1, p.80-89, 1991.

HOLMANN, F.; BLAKE, R.W.; BARKER, R.; MILLIGAN, R.A.; OLTENACU, P.A.; STANTON, T. L. Comparative profitability of purebred and crossbreed Holstein herds in Venezuela. Journal of Dairy Science, Savoy, v.73, n.8, p.2190-2205, 1990.

MONARDES, H.G.; ALMEIDA, R.; RIBAS, N.P. Estudo da idade ao primeiro parto em vacas da raça Holandesa, região Batavo, Paraná. In: REUNIÃO ANUAL DA SOCIEDADE BRASILEIRA DE ZOOTECNIA, 32, 1995, Brasília. Anais... Brasília: SBZ, 1995. p.665-70. 
Fatores ambientais sobre a idade ao primeiro parto, dias abertos e intervalo entre partos em vacas da raça...

PEREIRA, I.G.; GONÇALVES, T.M.; OLIVEIRA, A.I.G.; TEIXEIRA, N.M. Fatores de variação e parâmetros genéticos dos períodos de serviço e seco em bovinos da raça holandês de Minas Gerais. Revista da Sociedade Brasileira de Zootecnia, Viçosa, v.29, n.4, p.1005-1013, 2000.

PIMPÃO, C.T. Estudo de características produtivas e reprodutivas em rebanhos Holandeses da região de Arapoti, no Estado do Paraná. Curitiba, 1996. 107 f. Dissertação (Mestrado em Ciências Veterinárias) - Setor de Ciências Agrárias, Universidade Federal do Paraná.

POLASTRE, R.; MILAGRES, J.C.; TEIXEIRA, N.M.; CASTRO, C.A. Fatores genéticos e de ambiente no desempenho de vacas mestiças Holandês-Zebu: I. idade ao primeiro parto. Revista da Sociedade Brasileira de Zootecnia, Viçosa, v.16, n.3, p.227-32, 1987.

PROGRAMME D'ANALYSE DES TROUPEAUX LAITEIERS DU QUÉBEC - PATLQ. Rappor de Production 2000 du PATLQ, Québec, Canadá. Le Producteur de Lait Quebecóis, numéro spécial, v.21, 2001.

QUEIROZ, S.A.; GIANNONI, M.A.; RAMOS, A.A.; JONHATI, $H$. Environmental effects on the variation of productive traits in Holstein-Friesian $\times$ Zebu crossbreed cattle in the region of São Carlos, Brazil: I milk yield. Revista Brasileira de Genética, São Carlos, v.10, n.1, p.63-73, 1987.

RIBAS, N.P.; ALMEIDA, R.; HORST, J.A.; GIACOMAZZI, M.V. Período de serviço em rebanhos da raça Holandesa no Estado do Paraná. Revista Batavo, Carambeí, n.70, p.13-16, 1997b.

RIBAS, N.P.; ALMEIDA, R.; PIMPÃO, C.T.; RITCHER, G.O. Estudo da idade ao primeiro parto em rebanhos da raça Holandesa no Estado do Paraná. Revista Batavo, Carambeí, n.67, p.46-48, 1997a.

RIBAS, N.P.; MILAGRES, J.C.; SILVA, M.A.; CASTRO, A.C.G. Estudo da idade ao primeiro parto e intervalo entre partos em rebanhos Holandeses da bacia leiteira de Castrolanda, Estado do Paraná. Revista da Sociedade Brasileira de Zootecnia, Viçosa, v.12, n.4, p.756-60, 1983.
RICHTER, G.O. Estudo de características produtivas e reprodutivas em rebanhos da raça holandesa na região de Witmarsum, Paraná. Curitiba, 1995. 68 f. Dissertação (Mestrado em Ciências Veterinárias) - Setor de Ciências Agrárias, Universidade Federal do Paraná.

RORATO, P.R.N. Interação genótipo-ambiente no desempenho produtivo de vacas da raça Holandesa. Ribeirão Preto, 1988. Tese (Doutorado) - Faculdade de Medicina de Ribeirão Preto, Universidade de São Paulo.

SANTOS, J.E.P. Impactos da nutrição sobre a reprodução de bovinos. In: CONGRESSO BRASILEIRO DE RAÇAS ZEBUÍNAS: A INTEGRAÇÃO DA CADEIA PRODUTIVA, 3., 1998, Uberaba. Anais... Uberaba, 1998. p.24-76.

SAS $₫$ System for Linear Models. 3 ed. SAS. Institute Inc., Cary, NC, Estados Unidos, 1991.

SILVA, H.M.; WILCOX, C.J.; THATCHER, W.N.; BECKER, R.B.; MORSE, D. Factors affecting days open, gestation length and calving interval in Florida dairy cattle. Journal of Dairy Science, Savoy, v.75, p.288-293, 1992.

SIMERL, N.A ; WILCOX, C.J.; THATCHER, W.N. Postpartum performance of dairy heifers freshening at young ages. Journal of Dairy Science, Savoy, v.75, n.2, p.590-95, 1992.

VÁSQUEZ, E.F.A.; HERRERA, A.P.N. Concentração plasmática de cortisol, uréia, cálcio e fósforo em vacas de corte mantidas a pasto suplementadas com levedura de cromo durante a estação de monta. Ciência Rural, Santa Maria, v.33, n.4, 2003. Disponível em: <http://www.scielo.br>. Acesso em: 04 dez. 2003.

ZARNECKI, A.; JAMROZIK, J.; NORMAN, H.D. Comparision of tem Friesian strains in Poland for yield traits from first three parities. Journal of Dairy Science, Savoy, v.74, n.7, p.2303-08, 1991.

ZOCCAL, R. Leite em números: o agronegócio do leite no Brasil. Juiz de Fora: Embrapa Gado de Leite, 2001.

Recebido para publicação: $\quad$ 03/03/2004 Aprovado:

$04 / 07 / 2004$ 